\title{
Brain mapping: a novel intraoperative neuropsychological approach
}

\author{
Miran Skrap, MD, ${ }^{1}$ Dario Marin, PsyD, ${ }^{2}$ Tamara lus, MD, PhD, ${ }^{1}$ Franco Fabbro, MD, PhD, ${ }^{2}$ and \\ Barbara Tomasino, $\mathrm{PhD}^{2}$

\begin{abstract}
'Unità Operativa di Neurochirurgia, A.O.S. Maria della Misericordia, Udine; and ${ }^{2}$ RCCS "E. Medea," San Vito al Tagliamento (PN), Italy
\end{abstract}

\begin{abstract}
OBJECTIVE Awake surgery and mapping are performed in patients with low-grade tumors infiltrating functional brain areas for which the greater the resection, the longer the patient survival. However, the extent of resection is subject to preservation of cognitive functions, and in the absence of proper feedback during mapping, the surgeon may be less prone to perform an extensive resection. The object of this study was to perform real-time continuous assessment of cognitive function during the resection of tumor tissue that could infiltrate eloquent tissue.
\end{abstract}

METHODS The authors evaluated the use of new, complex real-time neuropsychological testing (RTNT) in a series of 92 patients. They reported normal scoring and decrements in patient performance as well as reversible intraoperative neuropsychological dysfunctions in tasks (for example, naming) associated with different cognitive abilities.

RESULTS RTNT allowed one to obtain a more defined neuropsychological picture of the impact of surgery. The influence of this monitoring on surgical strategy was expressed as the mean extent of resection: $95 \%$ (range $73 \%-100 \%$ ). At 1 week postsurgery, the neuropsychological scores were very similar to those detected with RTNT, revealing the validity of the RTNT technique as a predictive tool. At the follow-up, the majority of neuropsychological scores were still $>70 \%$, indicating a decrease of $<30 \%$.

CONCLUSIONS RTNT enables continuous enriched intraoperative feedback, allowing the surgeon to increase the extent of resection. In sharp contrast to classic mapping techniques, RTNT allows testing of several cognitive functions for one brain area under surgery.

http://thejns.org/doi/abs/10.3171/2015.10.JNS15740

KEY WORDS glioma; brain mapping; cognitive functions; neuropsychology; awake surgery; surgical technique

$\mathrm{D}$ IRECT electrical stimulation (DES) mapping is a well-known technique for identifying brain functions in an eloquent brain area during surgery., 3,8 , $13,16,26,27,29,40$ It can minimize permanent postoperative deficits while maximizing the extent of resection, especially in cases of infiltrative tumors such as low-grade gliomas (LGGs), in which the greater the resection, the longer the patient survival. . $^{9} 10,19,20,25,29,34,35$

In routine mapping, DES usually provides a high percentage of positive responses for relatively simple functions in sensorimotor areas at both cortical and subcortical levels $^{3,11,12,18,29}$ and for simple language tasks such as counting. For more complex tasks such as object naming, ${ }^{3,11,12,18,29}$ as well as tasks testing extralinguistic functions, the inter- pretation of DES effects is more complex. The percentage of positive responses significantly decreases for extralinguistic tasks, especially in subcortical mapping and in nondominant-hemisphere mapping, which, in general, are performed in rare cases only. . $14,15,17,21,32,32,41,44^{2}$

Preoperative diffusion tensor imaging (DTI) serves as orientation during surgical planning, but intraoperative confirmation with DES in the white matter is needed. However, DES does not offer the same guarantee of response in the white matter as in the cortex. In fact, few studies have reported the exact percentage of positive responses during subcortical DES for critical-function mapping outside the sensorimotor areas or Broca's area. . $10,20,22^{2}$

Consequently, the concept of "negative mapping" was

ABBREVIATIONS DES = direct electrical stimulation; DTI = diffusion tensor imaging; EOR = extent of resection; fMRI = functional MRI; LGG = low-grade glioma; MEP = motor evoked potential; RTNT = real-time neuropsychological testing; SSEP = somatosensory evoked potential.

SUBMITTED April 1, 2015. ACCEPTED October 19, 2015.

INCLUDE WHEN CITING Published online February 5, 2016; DOI: 10.3171/2015.10.JNS15740. 
defined owing to possible low DES positivity. ${ }^{35}$ Several factors could be responsible for negative mapping, such as a smaller craniotomy, individual variability, anatomical distortions, functional reorganization due to a tumor mass, an inappropriate task for the target area, and a network of areas subserving cognitive functioning that cannot be affected by the small area where DES exerts its electrical interference. ${ }^{28}$

Thus, in the absence of DES-induced responses, the surgeon cannot rely on proper feedback during the procedure and may be less prone to perform an extensive resection, even if negative mapping is correlated with a better clinical result..$^{35}$ The lack of positive DES responses does not necessarily imply a lack of function in the target area; therefore, the patient could experience postoperative dysfunction. Because of the possible dissonance between intraoperative mapping and postoperative outcome and in order to obtain a real-time view of what happens to cognitive functioning during the resection of tumor tissue that may infiltrate functionally preserved tissue, we used a novel approach consisting of more complex, continuous real-time neuropsychological testing (RTNT) performed during tumor resection.

While DES gives on/off responses on a relatively few simple tasks, RTNT offers supplementary information about different cognitive functions. Moreover, the resection can be continued without feedback between 2 DES phases. As compared with the DES used in the past, RTNT offers continuous feedback. In addition, it is likely to trigger the functioning of larger networks and thus reflects a dynamic view of brain functions. It also reduces the uncertainty associated with negative mapping and the temporal gaps between sequential DES phases throughout a resection. Furthermore, in RTNT many tasks can be continuously rotated, while DES allows testing of just a few simple tasks because of time constraints. Lastly, RTNT allows assessment of several cognitive functions for a particular brain area in surgery, while only 1 function can be tested during DES.

In this study, we present our experience with continuous RTNT during surgery for LGGs. We describe patient performance during surgery, reversible intraoperative neuropsychological dysfunction or oscillations, the influence of such monitoring on surgical strategy, and clinical and surgical outcomes.

\section{Methods \\ Patients}

RTNT was performed in a consecutive series of patients (and is still used at our institution). The criteria we used to perform RTNT are the same as those used for awake surgery. However, since RTNT is demanding, which can be one of the limitations of this technique, we used an additional inclusion criterion: presurgical neuropsychological performance should be $>85 \%$ in terms of accuracy.

We selected 92 patients -65 subjects with low-grade gliomas, 23 with anaplastic gliomas, and 4 with cavernomas-all right-handed, native Italian speakers (Table 1). The 4 patients with cavernomas served as a comparative group.
The local ethics committee approved the study. All patients gave written consent.

\section{Presurgical Evaluation}

Seven to 10 days prior to craniotomy, all patients underwent diagnostic evaluation including functional MRI (fMRI), anatomical and diffusion tensor imaging (DTI) as previously described, ${ }^{42,43}$ and neuropsychological testing (Supplementary Table S1).

\section{Surgery}

All patients underwent awake surgery as previously described. ${ }^{19}$ All patients were fixed to a Mayfield frame and remained awake from the beginning of the surgery. They were slightly sedated during exposure and closure of the surgical field..$^{19,38}$ Anatomical imaging, fMRI, and DTI data were sent to the navigator system (StealthStation, Medtronic), and they proved particularly useful during cortical mapping immediately after opening the dura mater. Standard tags were used to identify functions on the brain surface. Guided by the navigator, we placed colored tags along the medial border of the lesion through the tumor mass for spatial orientation during the advanced stage of tumor resection, when brain shifts usually occur. Surgery was performed under cortical and subcortical white matter DES, according to the intraoperative technique previously described by Duffau et al. ${ }^{10}$ and based on the methodology of Berger and Ojemann..$^{2,4,29,30}$ Motor evoked potentials (MEPs) and somatosensory evoked potentials (SSEPs) were also recorded during surgery to continuously monitor the integrity of motor and somatosensory pathways (64-channel Eclipse Neurovascular Workstation, Axon Systems Inc.; 32-channel video polygraphic station, Brain Quick SystemPlus, MicroMed). Continuous electrocorticography was performed to detect discharge phenomena and rule out the possibility that the patient's decrement in performance or oscillatory performance was caused by the concomitant presence of short focal seizures.

\section{Surgical Strategy and RTNT and DES}

Before resection, DES was performed as described in the literature. RTNT was started at the beginning of the resection and ended at the beginning of hemostasis. During surgery, at intervals, DES was performed in the classic way. In such cases we temporarily stopped the RTNT. If DES evoked a positive effect, the surgeon usually stopped the resection. If DES produced a negative response, we restarted the RTNT, which remained the only feedback on the patient's performance and was evaluated by the neuropsychologist.

The battery of tasks included in RTNT was created as follows. Tasks were selected from published neuropsychological batteries available in the Italian normative data. We included tasks encompassing a wide range of cognitive functions to have an exhaustive intraoperative neuropsychological battery. Some tasks are common for both right- and left-hemisphere lesions, testing 1) orientation, 2) memory and attention, 3) automatic series, and 4) fluency (Supplementary Table S1). For patients with left- 
TABLE 1. Summary of demographic and tumor details in 92 patients who underwent RTNT

\begin{tabular}{|c|c|c|c|c|}
\hline Parameter & LGG & Anaplastic Glioma & Cavernoma & Total \\
\hline No. of cases & 65 & 23 & 4 & 92 \\
\hline \multicolumn{5}{|l|}{ Sex } \\
\hline Male & 36 & 14 & 3 & 53 \\
\hline Female & 29 & 9 & 1 & 39 \\
\hline Mean age in yrs & $42.2 \pm 11.5$ & $51.98 \pm 15.12$ & $42.83 \pm 10.03$ & $45.67 \pm 13.18$ \\
\hline Mean education in yrs & $12.83 \pm 3.9$ & $11.57 \pm 2.58$ & $13.83 \pm 5$ & $12.74 \pm 3.91$ \\
\hline \multicolumn{5}{|l|}{ Side of tumor } \\
\hline $\mathrm{Lt}$ & 39 & 15 & 4 & 58 \\
\hline Rt & 26 & 8 & 0 & 34 \\
\hline \multicolumn{5}{|l|}{ Tumor location } \\
\hline Precentral & 29 & 12 & 1 & 42 \\
\hline Retrocentral & 8 & 4 & 1 & 13 \\
\hline Temporal & 10 & 5 & 1 & 16 \\
\hline Insular & 18 & 2 & 1 & 21 \\
\hline \multicolumn{5}{|l|}{ Symptoms at presentation } \\
\hline None, incidental finding & 3 & 0 & 0 & 3 \\
\hline Headache & 4 & 2 & 1 & 7 \\
\hline Neurological deficits & 5 & 3 & 0 & 8 \\
\hline Generalized seizure & 38 & 9 & 2 & 49 \\
\hline Motor seizure & 12 & 9 & 1 & 22 \\
\hline Viscerosensory seizure & 3 & 0 & 0 & 3 \\
\hline
\end{tabular}

hemisphere lesions, we used tasks testing 1) naming, 2) repetition, 3) reading, and 4) comprehension.

From our extensive list of tasks (Supplementary Table S1), we selected a series of tasks according to lesion localization, fMRI and DTI results, and preoperative neuropsychological profile. Task sequence follows a fixed order with regard to an area (Fig. 1). We created a fixed task order for lesions of the left frontal, left Broca's, left premotor, left temporoinsular, left parietal, right frontal, right premotor, right temporoinsular, and right parietal area. For example, for surgery involving the left temporal cortex, the sequence of RTNT includes object naming, phonemic discrimination, word reading, word repetition, nonword reading, nonword repetition, phonological discrimination, digit span, lexical decision, action naming, and picture description (Supplementary Table S2).

The sequence of tasks was repeated (presenting a different stimuli list for each sequence) until the end of the resection. Intraoperatively, however, task order and selection could suddenly change according to the principles of differential diagnosis (Supplementary Table S3). For example, in each test for a patient who underwent surgery in the left temporal lobe, 10-15 items were presented (for instance, 15 pictures for naming, 10 words for reading, 10 pseudowords for repetition) for about 30 seconds for each task, in a rotating manner. In this way task assessment and task switching were quick and dynamic for immediate dysfunction detection. As soon as the patient exhibited a decrement, the neurosurgeon was immediately informed and carried on with the surgical technique already described. In 1-2 minutes the RTNT allowed the assessment of object naming, word reading, and pseudoword repetition.
When this sequence was completed and if patient performance was within the normal range, we restarted testing using the first task and followed the same sequence but with different items. On the contrary, if a patient showed a decrement-for instance, if the patient's object naming performance decreased (in the example, the score was 10/15-anomia) -we performed in-depth testing. Following the principles of differential diagnosis (Supplementary Table S3), we assessed whether the decrease was confined to object naming, so we used action naming (in the example, the score was 10/15-anomia), reading of words and pseudowords (score 9/10 for each task), phonology (phonological discrimination: 15/16), picture description (showing that the anomies also impacted fluency: 2/4-anomia), lexicon (lexical decision: 18/20), and repetition (word: 10/10 and nonword: 10/10). We restarted with object naming (in the example, the score was 12/15-anomia) and action naming (13/15-anomia), picture description (3/4-anomia), digit span (5/9), and lexical decision (19/20), and we again administered object naming. If the performance was restored (14/15) we continued with a second sequence of the RTNT phase (Supplementary Table S3). This protocol was, of course, subject to real surgical timing. If everything runs smoothly, a sequence like the one described lasts about 7-8 minutes.

The possible deterioration in performance usually occurred in a gradual way. An interesting feature was the often temporary nature of the deterioration, with a recovery in performance after some minutes (Fig. 2). Considering that tissue traumatism due to manipulation or patient fatigue may have caused this oscillation, we adopted a more dynamic surgical strategy. To assess the effect of surgical 


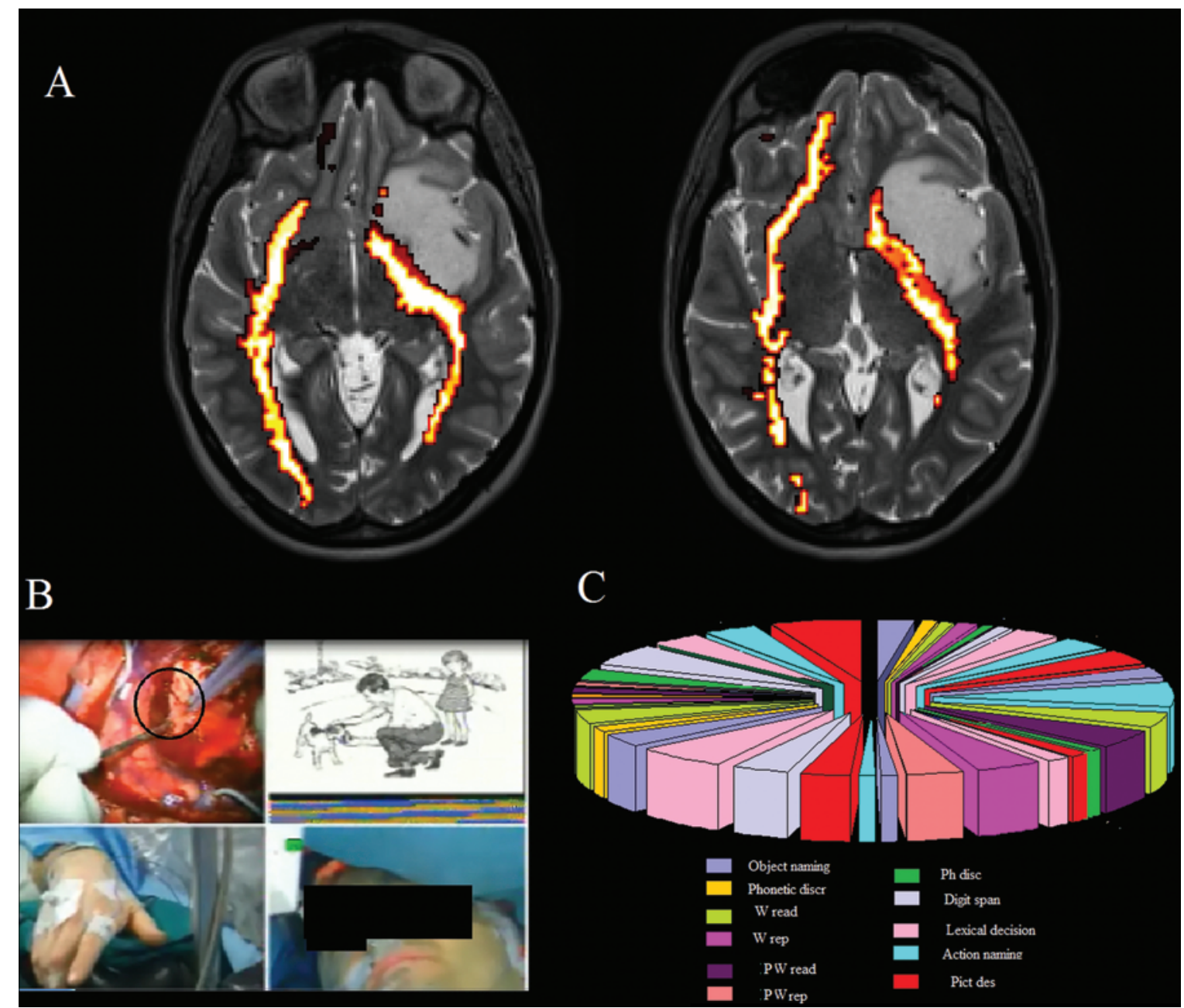

FIG. 1. Case 12. Images obtained in a patient with an LGG in the temporoinsular area with the left inferior frontooccipital fasciculus passing close to the lesion (A). The patient performed RTNT (B). Black circle indicates the point of manipulation where the patient exhibited oscillatory performance, that is, decreased performance followed by recovery. The sequence of tasks was rotated during resection (C); note the repetition of a sequence of tasks (same color, same test). Figure is available in color online only.

manipulation, we briefly interrupted the surgery, giving the patients short breaks, or we moved to another part of the surgical field by using the "stop-and-go" surgical approach as done for intramedullary tumors. ${ }^{3}$ With this approach, patients were able to rest and restart performing the tasks, and we were able to continue the procedure in the same area if the patients recovered.

Using the logic of neuropsychological differential diagnosis with different tests, we could either confirm or exclude that testing was affected by manipulation or fatigue. This is fairly similar to standard neuropsychological testing outside the operating room, when fatigue can affect patient performance as well.

The limit used to stop surgery temporarily or definitively coincided with a decrease in performance to $70 \%$ of baseline levels. The $30 \%$ threshold was established by examining a preliminary group of patients in whom we tested the feasibility of our technique before actually starting the present study. Thus this threshold was correlated to follow-up performance. For example, for the object naming task, 1) we identified patients with pathology at the follow-up examination and calculated their mean performance, which was around 75\%; and 2) we identified their RTNT level of performance on the same task and calculated their mean accuracy, which was around $70 \%$. Consequently, we considered this level of performance as a warning to stop surgery. In the present series of patients, we set the threshold at the same value on the basis of our initial experience and verified whether it worked by correlating RTNT performance with the follow-up performance.

Deterioration usually occurred when the resection was very close to the tumor boundary. The above-described strategy provided us with ongoing confirmation that we could continue with a resection. In contrast, with a negative DES response, we lacked the confidence necessary to continue with an aggressive and somewhat blind surgery in an eloquent area. DES in the same areas did not produce a similar response. During RTNT, each subject viewed the stimuli (Presentation, Neurobehavioral Systems Inc.) via 
A Object Naming RTNT Accuracy

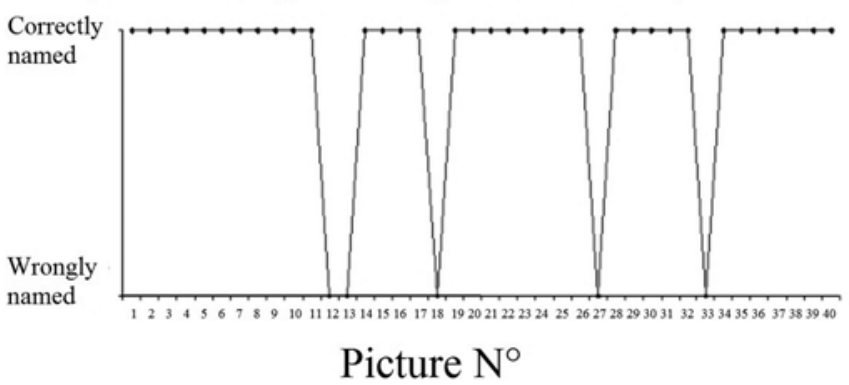

B

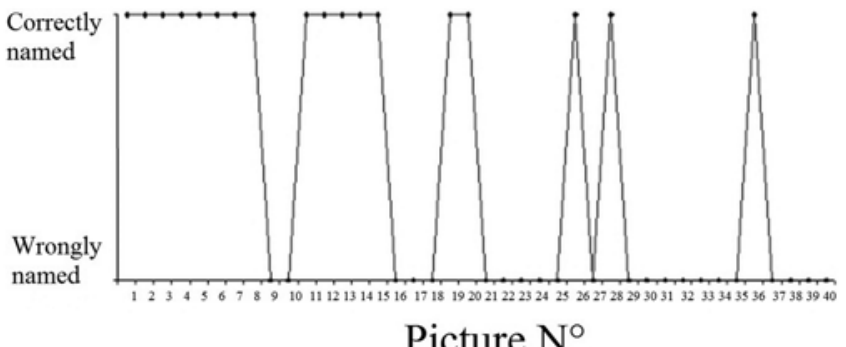

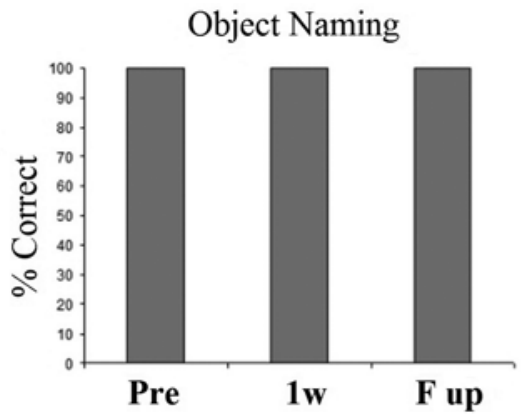

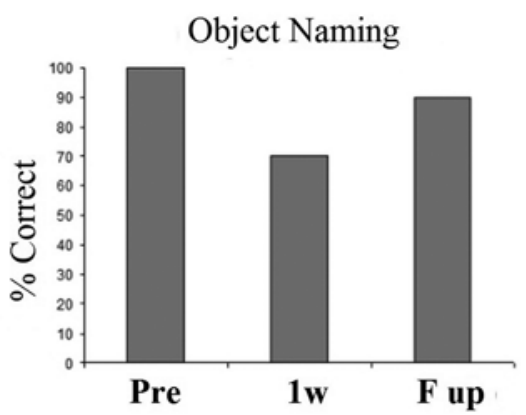

FIG. 2. Cases 4 and 21. Two different patterns of performance during object naming, in which the patients either named the items correctly or did not. In the patient in Case 21 (A left), the deterioration was often just temporary, with recovery in the impaired performance after some minutes (reversible neuropsychological deficit), whereas in the patient in Case 4 (B left), the decrement was more stable. These patterns were confirmed by neuropsychological testing at 1 week (1w) after surgery and at follow-up ( $F$ up), showing no decrement in the patient in Case 21 (A right) as compared with a temporary decrement in the patient in Case 4 (B right) at 1 week after surgery. Pre = presurgery.

a monitor at a distance of $30 \mathrm{~cm}$. Audio from the patients and the neuropsychologist was recorded. The intraoperative information has provided feedback to the surgeon.

\section{Statistical Analysis}

Nonparametric statistics were applied (Wilcoxon test, 2-tailed, $\alpha$ level $<0.05$ ) to compare the patient's accuracy pre- versus intraoperatively, preoperatively versus 1 week postoperatively, and preoperatively versus follow-up.

\section{Results}

\section{Postsurgical Evaluation}

All patients underwent standard diagnostic MRI with tumor volume analysis ${ }^{19}$ and neuropsychological testing.

\section{Neuropsychological Performance, RTNT, and Follow-Up}

To determine each patient's performance accuracy, the reference level $(100 \%)$ was set at his or her preoperative performance. Scores were thus normalized. To correlate the impact of each test on the follow-up level of performance, we grouped the tests into 3 categories: Group 1, absent or minimal intraoperative dysfunction with complete recovery at the end of surgery; Group 2, intraoperative dysfunction still present at 1 week after surgery and complete recovery achieved 6 months after surgery; and Group 3, intraoperative dysfunction and some deficits at 6 months after surgery.

At 1 week postsurgery, scores were very similar to those detected with RTNT, proving the validity of the RTNT technique as a predictive tool. The majority of scores were still $>70 \%$ of the baseline performance, which indicated a decrease $<30 \%$. For example, for a critical function such as naming, significant improvement was found in the lefthemisphere LGG group at follow-up (from $79 \%$ at 1 week postsurgery to $86 \%$ at the 6-month follow-up). Results of tests that were the most critical in our sample appear in Tables 2-6.

\section{Task Sequence and Duration}

Mean duration of testing during the resection was $43 \pm$ 21 minutes, and the mean number of tasks administered was $16 \pm 21$. A mean of $3.9 \pm 1.4$ tasks were administered during 10 minutes. For example, as can be seen in Supplementary Table S3, the total time needed for resection of an LGG in the left temporal lobe was 55.40 minutes.

\section{Comparative Study With a Cavernoma Group}

The patients with a cavernoma had very high performance scores (Table 4). The cavernomas were localized according to presurgical fMRI in language-related areas at both cortical and intrasulcal levels. Lesion size ranged from 12 to $22 \mathrm{~cm}^{3}$ including the hemosiderin layer. Localization was the left insula, postcentral area, and precentral area. Two patients had generalized seizures, 1 patient had motor seizures, and 1 patient had headaches. In all 4 cases but 1 , the thin layer of hemosiderin was removed ac- 


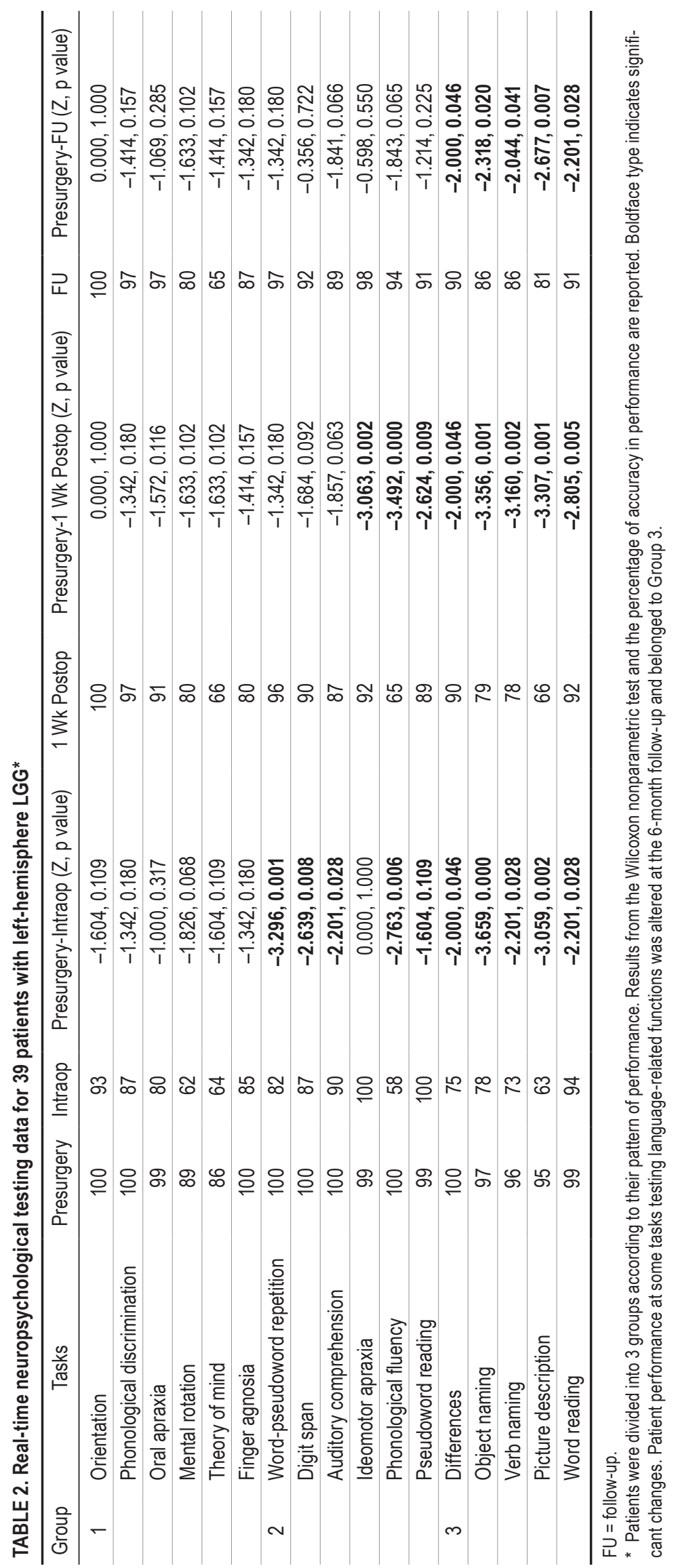


TABLE 3. Real-time neuropsychological testing data for 15 patients with left-hemisphere anaplastic glioma*

\begin{tabular}{clccccc}
\hline Group & \multicolumn{1}{c}{ Real-Time Tasks } & Preop & Intraop & Preop-Intraop (Z, p value) & 1 Wk & Preop-1 Wk (Z, p value) \\
\hline 1 & Oral apraxia & 99 & 100 & $0.000,1.000$ & 97 & $-1.069,0.285$ \\
\hline & Ideomotor apraxia & 93 & 100 & $-1.000,0.317$ & 91 & $-0.507,0.612$ \\
\hline & Orientation & 100 & 87 & $-1.342,0.180$ & 100 & $0.000,1.000$ \\
\hline & Phonological discrimination & 100 & 72 & $-1.414,0.157$ & - & - \\
\hline & Auditory comprehension & 100 & 90 & $-1.604,0.109$ & - & - \\
\hline & Object naming & 90 & 70 & $-1.362,0.173$ & $-0.774,0.439$ & $-0.447,0.655$ \\
\hline & Digit span & 100 & 93 & $-1.633,0.102$ & 93 & $-1.069,0.285$ \\
\hline & Repetition & 99 & 84 & $-1.461,0.144$ & 87 & $-0.405,0.686$ \\
\hline & Word Reading & 96 & 78 & $-1.604,0.109$ & 95 & $-0.535,0.593$ \\
\hline
\end{tabular}

* Patients were divided in 2 groups according to their pattern of performance. Results from the Wilcoxon nonparametric test and the percentage of accuracy in performance are reported. Boldface type indicates the significant changes detected between pre- and intraoperative testing, and preoperative and 1-week testing. The followup ended at 1 week since later anaplastic patients received radio- or chemotherapy, which can worsen cognitive functioning. For this reason, we avoided adding an additional variable in our patient series. We decided to exclusively focus on RTNT performance correlated to resection and to follow up performance for those patients for whom no other uncontrollable variables (such as the cognitive decline related to chemotherapy and radiation) were present at the follow-up testing.

cording to negative DES and RTNT performance. In that 1 case, the DES response was positive in the hemosiderin layer and thus we left that layer.

Since cavernomas are extraaxial lesions not infiltrating the parenchyma as gliomas do, the comparison with cavernoma patients served as a validation study and enabled us to conclude that RTNT is reliable for measuring intraoperative cognitive changes, as the patients could perform the RTNT with a high degree of proficiency.

\section{Impact on Resection}

We compared the value of the extent of resection (EOR) based on our intraoperative protocol with that using our historical database. ${ }^{19}$ As reported in our previous study, ${ }^{19}$ patients in Series 1 (those who had undergone intraoperative DES alone) had a mean EOR of $77 \%$ (range 28\%-
$100 \%$ ) and patients in Series 2 (those who had undergone intraoperative DES plus had overlapping fMRI/fiber tracking DTI data on a neuronavigation system) had a mean EOR of $90 \%$ (range $49 \%-100 \%$ ), whereas patients in the present study had a mean EOR of 95\% (range 73\%-100\%).

\section{Discussion \\ Mapping and Functions}

Maximal resection is currently the first therapeutic option for LGGs to prolong overall survival. ${ }^{19,24,34,36,38,39}$ Given the ability of LGGs to infiltrate eloquent cortical areas and subcortical functional pathways, ${ }^{19,23}$ brain mapping techniques and awake surgery have become mandatory in dealing with such lesions. ${ }^{3,9,10,19,20,35}$ In contrast to the situation for cortical DES, only a few studies concerning language function have documented in detail the per-

TABLE 4. Real-time neuropsychological testing performance of 4 patients with left-hemisphere cavernoma*

\begin{tabular}{clrrrrrrr}
\hline Group & Real-Time Tasks & Preop & Intraop & Preop-Intraop (Z, p value) & 1 Wk & Preop-1 Wk (Z, p value) & FU & Preop-FU (Z, p value) \\
\hline 1 & 100 & 100 & $0.000,1.000$ & 100 & $0.000,1.000$ & 100 & $0.000,1.000$ \\
\hline & Orientation & 100 & 99 & $-1.000,0.317$ & 98 & $-1.000,0.317$ & 98 & $-1.000,0.317$ \\
\hline Object naming & 100 & 100 & $0.000,1.000$ & 100 & $0.000,1.000$ & 100 & $0.000,1.000$ \\
\hline Word reading & 100 & 100 & $0.000,1.000$ & 100 & $0.000,1.000$ & 100 & $0.000,1.000$ \\
\hline Pseudoword reading & 97 & 97 & $0.000,1.000$ & 99 & $0.000,1.000$ & 99 & $0.000,1.000$ \\
\hline Oral apraxia & 100 & 100 & $0.000,1.000$ & 100 & $0.000,1.000$ & 100 & $0.000,1.000$ \\
\hline Repetition & 100 & 90 & $-1.000,0.317$ & 95 & $-1.000,0.317$ & 100 & $-1.000,0.317$ \\
\hline Ideomotor apraxia & 100 & 100 & $0.000,1.000$ & 100 & $0.000,1.000$ & 100 & $0.000,1.000$ \\
\hline Word writing & 100 & 100 & $0.000,1.000$ & 100 & $0.000,1.000$ & 100 & $0.000,1.000$ \\
\hline Pseudoword writing & 100 & 120 & $-1.342,0.180$ & 94 & $-1.000,0.317$ & 101 & $-0.447,0.655$ \\
\hline Digit span & 100 & 100 & $-1.000,0.317$ & 100 & $-1.000,0.317$ & 100 & $0.000,1.000$ \\
\hline Picture description & 100 & 80 & $-1.000,0.317$ & 80 & $-1.000,0.317$ & 105 & $-0.447,0.655$ \\
\hline Digit span backward & 100 & 105 & $-1.342,0.180$ & 103 & $-1.342,0.180$ & 130 & $-1.342,0.180$ \\
\hline Digit span & 100 &
\end{tabular}

\footnotetext{
* Patients were divided in 3 groups according to their pattern of performance. Results from the Wilcoxon nonparametric test and the percentage of accuracy in performance are reported. No significant changes were detected between pre- and intraoperative testing, preoperative and 1-week testing, and preoperative and follow-up (at 6 months) testing. All tasks were unaltered at 1 week and at 6 months and belonged to Group 1.
} 
TABLE 5. Real-time neuropsychological testing data for 26 patients with right-hemisphere LGG*

\begin{tabular}{|c|c|c|c|c|c|c|c|c|}
\hline Group & Real-Time Tasks & Preop & Intraop & Preop-Intraop (Z, p value) & $1 \mathrm{Wk}$ & Preop-1 Wk (Z, p value) & FU & Preop-FU (Z, p value) \\
\hline \multirow[t]{11}{*}{1} & Orientation & 100 & 98 & $-1.000,0.317$ & 97 & $-1.000,0.317$ & 100 & $0.000,1.000$ \\
\hline & Stroop & 100 & 100 & $0.000,1.000$ & 100 & $-1.000,0.317$ & 100 & $0.000,1.000$ \\
\hline & Automatic series & 100 & 100 & $0.000,1.000$ & 100 & $0.000,1.000$ & 100 & $0.000,1.000$ \\
\hline & Reading dyslexia & 100 & 95 & $-1.342,0.180$ & 96 & $-1.000,0.317$ & 100 & $0.000,1.000$ \\
\hline & Picture description & 100 & 100 & $0.000,1.000$ & 100 & $0.000,1.000$ & 100 & $0.000,1.000$ \\
\hline & Calculation & 100 & 97 & $-1.604,0.109$ & 96 & $-1.732,0.083$ & 96 & $-1.732,0.083$ \\
\hline & Target detection & 99 & 96 & $-0.447,0.655$ & 96 & $-1.342,0.180$ & 97 & $-1.000,0.317$ \\
\hline & Digit span backward & 100 & 85 & $-1.000,0.317$ & 82 & $-1.342,0.180$ & 100 & $-1.342,0.180$ \\
\hline & Digit span & 100 & 91 & $-1.342,0.180$ & 103 & $-1.000,0.317$ & 95 & $-1.342,0.180$ \\
\hline & Line bisection & 100 & 96 & $0.000,1.000$ & 83 & $-1.000,0.317$ & 100 & $0.000,1.000$ \\
\hline & Clock test & 91 & 90 & $-0.272,0.785$ & 90 & $-1.342,0.180$ & 92 & $-1.633,0.102$ \\
\hline \multirow[t]{3}{*}{2} & Stars cancellation & 95 & 97 & $-0.447,0.655$ & 97 & $-2.014,0.044$ & 99 & $-0.213,0.832$ \\
\hline & $\begin{array}{l}\text { Handedness decision/men- } \\
\text { tal rotation }\end{array}$ & 100 & 92 & $-2.023,0.043$ & 92 & $-2.023,0.043$ & 96 & $-1.414,0.157$ \\
\hline & Metaphor comprehension & 94 & 77 & $-2.803,0.005$ & 81 & $-2.375,0.018$ & 88 & $-1.890,0.059$ \\
\hline
\end{tabular}

* Patients were divided in 3 groups according to their pattern of performance. Results from the Wilcoxon non-parametric test and the percentage of accuracy in performance are reported. Boldface type indicates the significant changes detected between pre- and intraoperative testing, preoperative and 1-week testing, and preoperative and follow-up testing. None of the tests belonged to Group 3, meaning that patients had completely recovered at the 6-month follow-up testing.

centage of positive responses for subcortical $\mathrm{DES}^{1,10,20}$ and extralinguistic functions. ${ }^{7,14,15,17,21,31,32,41,44}$ Consequently, the concept of negative mapping was proposed even if, in the absence of a DES effect, impaired cognitive performance was found at follow-up neuropsychological testing. ${ }^{5}$

\section{Real-Time Neuropsychological Testing and Surgery}

Nowadays we accept that the relation between one brain function and a brain area is not 1:1 and, instead, that cognitive processing is subserved by multiple brain networks. Consequently, the brain areas involved in cognitive processing are larger than the small, circumscribed portion of brain on which DES exerts its electrical interference.
Accordingly, RTNT, which is complementary to DES, enables the surgeon to monitor a patient's cognitive performance, reducing the uncertainty associated with negative feedback on DES. A very interesting feature of RTNT, which significantly influenced our surgical strategy, was the oscillation in patient performance, with worsened and then recovered accuracy in a response within a few minutes. We considered this a warning signal, usually manifesting when the resection was close to the lesion border. When this happened, the resection was stopped and resumed in another site. If the patient's performance recovered, we returned to the same area and continued the resection after in-depth testing. Resection was stopped when

TABLE 6. Real-time neuropsychological testing data from 8 patients with right-hemisphere anaplastic glioma*

\begin{tabular}{|c|c|c|c|c|c|c|}
\hline Group & Real-Time Tasks & Preop & Intraop & Preop-Intraop (Z, p value) & $1 \mathrm{Wk}$ & Preop-1 Wk $(Z, p$ value $)$ \\
\hline \multirow[t]{12}{*}{1} & Orientation & 100 & 98 & $0.000,1.000$ & 100 & $0.000,1.000$ \\
\hline & Digit span & 100 & 100 & $0.000,1.000$ & 100 & $0.000,1.000$ \\
\hline & Reading dyslexia & 100 & 81.81 & $-1,0.317$ & 85 & $-1,0.317$ \\
\hline & Picture description & 100 & 83.6 & $-1.34,0.18$ & 100 & $0.000,1.000$ \\
\hline & Calculation & 100 & 87.2 & $-1,0.31$ & NE & NE \\
\hline & Target detection & 99 & 82.05 & $-0.447,0.655$ & 96 & $-1.342,0.180$ \\
\hline & Line bisection & 83.33 & 82.05 & $-0.44,0.65$ & 100 & $0.000,1.000$ \\
\hline & Star cancellation & 99.38 & 82.05 & $-1.6,0.102$ & 96.29 & $0.000,1.000$ \\
\hline & Clock test & 94.28 & 82.05 & $-1.47,0.141$ & 100 & $-1,0.31$ \\
\hline & Constructional apraxia & 91.96 & 82.05 & $-1.89,0.059$ & 92.85 & $0.000,1.000$ \\
\hline & Handedness decision/mental rotation & 85 & 65 & $-1,0.31$ & NE & NE \\
\hline & Metaphor comprehension & 92.5 & 74.61 & $-1.604,0.18$ & 80 & $-1.34,0.18$ \\
\hline
\end{tabular}

$\mathrm{NE}=$ not executed.

* Patients were divided in 2 groups according to their pattern of performance. Results from the Wilcoxon nonparametric test and the percentage of accuracy in performance are reported. No significant changes were detected. The follow-up ended at 1 week of testing since later anaplastic patients received radio- or chemotherapy. 
the patient's performance dropped to $70 \%$ of baseline levels. Importantly, we believe that the issue of the threshold should be carefully considered and, as in our group sample, could be highly dependent on several factors such as lesion localization, presurgical patient status, and the list of tasks used. In our series the 70\% threshold worked well, but we think that in the future in improving the battery of tasks, better analysis on the different tasks may influence the defined threshold. With RTNT feedback, the surgeon felt safer continuing the resection in comparison with cases when he had no feedback due to negative DES responses. Similar brain behavior has never been appreciable with DES, which, on the contrary, provides a simple on-off response to usually quite simple tests. Our results with RTNT led us to change the surgical procedure. In the present study, we did not operate on the same site for too long, preferring instead to change the surgical field quite often to temporarily interrupt the manipulation of brain tissue in the same site since we believed that allowing the structure to rest could facilitate the recovery of function. We might observe a similar reaction when traction is applied close to the corticospinal tract or in the medulla. Because of tissue manipulation, the MEPs and SSEPs could be temporarily altered or even disappear, although they could recover after a temporary interruption in the surgery.

In those cases in which DES was negative, the continuous feedback from the patient and the described surgical strategy allowed a larger resection. If compared with the simple responses of the 1-0 type evoked by DES, RTNT does not necessarily give stopping signals but does provide us with more information. We should mainly consider 2 reasons that have permitted increased resection. First, in the standard mapping technique, the negative response obtained with DES, at least in our experience, tends to inhibit major surgical aggressiveness. Without real feedback, the surgeon has simply stopped the resection earlier in apparently healthy tissue. A negative response in RTNT (that is, the patient performs within the normal range) is more informative than the negative response provided by DES (that is, negative mapping). Consequently, in the case of RTNT, the surgeon is less prone to stop resection and often is more confident in carrying it on. Second, in the present RTNT series, we learned that when a transient decrease happens, a recovery can occur after a short period of time (a few or several minutes) and consequently the resection can continue. This has induced us to adopt the stop-and-go technique. Applying the same logic to other fiber tracts, we would probably avoid a false-positive response that would stop surgery too early.

\section{Tests and Brain Areas}

Tests belonging to Group 3 might be seen as the most sensible tests for the most critical functions as they still indicated pathology at the follow-up. Most of them are correlated with language functions, as the patients' lesions mainly included the insular, temporal, and/or premotor areas. On the contrary, the tests involving extralinguistic functions on the dominant hemisphere as well as the nondominant hemisphere generally showed a complete recovery at 6 months after surgery. We also noticed a faster recovery in the group with right-hemisphere lesions. More selective tests may still be used in the future, but the need for awake craniotomy in areas different from the sensorimotor area on the nondominant side may be reconsidered. Clearly, these results are preliminary because they depend on the number of patients who performed a given task and on the lesion site. Nonetheless, this correlation could help determine the predictive value of RTNT. Overall, no task showed a preoperative versus a follow-up change $>25 \%$. As far as the neuropsychological follow-up at 6 months is concerned, with our detailed neuropsychological battery of tasks, we observed a decrease in performance mainly affecting language function that went unnoticed with standard neurosurgical evaluations.

\section{Clinical Results}

In this preliminary experience, continuous, more intensive, complex feedback allowed greater resection. From a mean EOR of $90 \%$ (range $49 \%-100 \%$ ) in our second historical series (that is, Series 2; Series 1 had a mean EOR of 77\% [range 28\%-100\%]), ${ }^{19}$ we reached a mean EOR of $95 \%$ in the present series (range $73 \%-100 \%$ ). It is in effect a modest increase, but several recent volumetric analyses have shown that even small improvements in the resection rate, such as from $>90 \%$ to $100 \%$, may provide an advantage both in terms of better overall survival and in terms of anaplastic transformation. $6,19,33,34,37$ More recent studies speak about supratotal resection in which a small difference in tumor resection can influence prognosis. ${ }^{6} \mathrm{In}$ principle, it is likely that even the modest improvement in the resection rate in our patient sample could lead to similar clinical advantages. Furthermore, we have to consider the qualitative aspect allowed by this method, which gives the surgeon better and safer feedback. In our opinion, it is difficult to resect infiltrated brain tissue despite negative DES mapping. Thus, it is likely that patients in the present RTNT series will have a different neuropsychological pattern at follow-up, as compared with other patients who did not undergo RTNT. By using this method, we could exert better tumor control and could better predict the patients' clinical follow-up. However, this series is still too small, and we need a greater number of cases to validate this resection method and evaluate its real impact on follow-up.

\section{Conclusions}

RTNT allows for ongoing monitoring of cognitive performance during resection of an infiltrative brain tumor in functional tissue. It enables one to evaluate cognitive functions during follow-up, obtaining a more defined neuropsychological picture of the impact of surgery. RTNT in combination with DES allows for continuous feedback, reducing the uncertainty of negative feedback on DES, particularly when operating in the white matter, and thus allowing one to increase the extent of resection.

\section{References}

1. Bello L, Gallucci M, Fava M, Carrabba G, Giussani C, Acerbi F, et al: Intraoperative subcortical language tract mapping guides surgical removal of gliomas involving speech areas. Neurosurgery 60:67-82, 2007

2. Berger MS: Lesions in functional ("eloquent") cortex and subcortical white matter. Clin Neurosurg 41:444-463, 1994 
3. Berger MS: Minimalism through intraoperative functional mapping. Clin Neurosurg 43:324-337, 1996

4. Berger MS, Ojemann GA: Intraoperative brain mapping techniques in neuro-oncology. Stereotact Funct Neurosurg 58:153-161, 1992

5. Bizzi A: Clinico-anatomical correlation in gliomas: a new frontier in clinical neuroscience? Cortex 47:1013-1015, 2011

6. Capelle L, Fontaine D, Mandonnet E, Taillandier L, Golmard JL, Bauchet L, et al: Spontaneous and therapeutic prognostic factors in adult hemispheric World Health Organization Grade II gliomas: a series of 1097 cases: clinical article. J Neurosurg 118:1157-1168, 2013

7. Desmurget M, Sirigu A: A parietal-premotor network for movement intention and motor awareness. Trends Cogn Sci 13:411-419, 2009

8. Duffau H: Lessons from brain mapping in surgery for lowgrade glioma: insights into associations between tumour and brain plasticity. Lancet Neurol 4:476-486, 2005

9. Duffau H, Capelle L, Denvil D, Sichez N, Gatignol P, Lopes M, et al: Functional recovery after surgical resection of low grade gliomas in eloquent brain: hypothesis of brain compensation. J Neurol Neurosurg Psychiatry 74:901-907, 2003

10. Duffau H, Capelle L, Denvil D, Sichez N, Gatignol P, Taillandier L, et al: Usefulness of intraoperative electrical subcortical mapping during surgery for low-grade gliomas located within eloquent brain regions: functional results in a consecutive series of 103 patients. J Neurosurg 98:764-778, 2003

11. Duffau H, Capelle L, Sichez JP, Bitar A, Faillot T, Arthuis F, et al: [Surgery of the central nervous system: value of preoperative functional brain mapping by direct electric stimulation.] Presse Med 29:421-422, 2000 (Fr)

12. Duffau H, Capelle L, Sichez N, Denvil D, Lopes M, Sichez JP, et al: Intraoperative mapping of the subcortical language pathways using direct stimulations. An anatomo-functional study. Brain 125:199-214, 2002

13. Duffau H, Moritz-Gasser S, Mandonnet E: A re-examination of neural basis of language processing: proposal of a dynamic hodotopical model from data provided by brain stimulation mapping during picture naming. Brain Lang 131:1-10, 2014

14. Fernández Coello A, Duvaux S, De Benedictis A, Matsuda $\mathrm{R}$, Duffau H: Involvement of the right inferior longitudinal fascicle in visual hemiagnosia: a brain stimulation mapping study. J Neurosurg 118:202-205, 2013

15. Gharabaghi A, Fruhmann Berger M, Tatagiba M, Karnath HO: The role of the right superior temporal gyrus in visual search-insights from intraoperative electrical stimulation. Neuropsychologia 44:2578-2581, 2006

16. Gil-Robles S, Carvallo A, Jimenez MdelM, Gomez Caicoya A, Martinez R, Ruiz-Ocaña C, et al: Double dissociation between visual recognition and picture naming: a study of the visual language connectivity using tractography and brain stimulation. Neurosurgery 72:678-686, 2013

17. Giussani C, Pirillo D, Roux FE: Mirror of the soul: a cortical stimulation study on recognition of facial emotions. J Neurosurg 112:520-527, 2010

18. Ilmberger J, Ruge M, Kreth FW, Briegel J, Reulen HJ, Tonn JC: Intraoperative mapping of language functions: a longitudinal neurolinguistic analysis. J Neurosurg 109:583-592, 2008

19. Ius T, Isola M, Budai R, Pauletto G, Tomasino B, Fadiga L, et al: Low-grade glioma surgery in eloquent areas: volumetric analysis of extent of resection and its impact on overall survival. A single-institution experience in 190 patients: clinical article. J Neurosurg 117:1039-1052, 2012

20. Keles GE, Lundin DA, Lamborn KR, Chang EF, Ojemann G, Berger MS: Intraoperative subcortical stimulation mapping for hemispherical perirolandic gliomas located within or adjacent to the descending motor pathways: evaluation of mor- bidity and assessment of functional outcome in 294 patients. J Neurosurg 100:369-375, 2004

21. Kleinman JT, Sepkuty JP, Hillis AE, Lenz FA, HeidlerGary J, Gingis L, et al: Spatial neglect during electrocortical stimulation mapping in the right hemisphere. Epilepsia 48:2365-2368, 2007

22. Maldonado IL, Moritz-Gasser S, de Champfleur NM, Bertram L, Moulinié G, Duffau H: Surgery for gliomas involving the left inferior parietal lobule: new insights into the functional anatomy provided by stimulation mapping in awake patients. J Neurosurg 115:770-779, 2011

23. Mandonnet E, Capelle L, Duffau H: Extension of paralimbic low grade gliomas: toward an anatomical classification based on white matter invasion patterns. J Neurooncol 78:179-185, 2006

24. McGirt MJ, Chaichana KL, Attenello FJ, Weingart JD, Than $\mathrm{K}$, Burger PC, et al: Extent of surgical resection is independently associated with survival in patients with hemispheric infiltrating low-grade gliomas. Neurosurgery 63:700-708, 2008

25. McGirt MJ, Chaichana KL, Gathinji M, Attenello FJ, Than $\mathrm{K}$, Olivi A, et al: Independent association of extent of resection with survival in patients with malignant brain astrocytoma. J Neurosurg 110:156-162, 2009

26. Moritz-Gasser S, Duffau H: The anatomo-functional connectivity of word repetition: insights provided by awake brain tumor surgery. Front Hum Neurosci 7:405, 2013

27. Moritz-Gasser S, Herbet G, Duffau H: Mapping the connectivity underlying multimodal (verbal and non-verbal) semantic processing: a brain electrostimulation study. Neuropsychologia 51:1814-1822, 2013

28. Nathan SS, Sinha SR, Gordon B, Lesser RP, Thakor NV: Determination of current density distributions generated by electrical stimulation of the human cerebral cortex. Electroencephalogr Clin Neurophysiol 86:183-192, 1993

29. Ojemann G, Ojemann J, Lettich E, Berger M: Cortical language localization in left, dominant hemisphere. An electrical stimulation mapping investigation in 117 patients. J Neurosurg 71:316-326, 1989

30. Ojemann GA: Functional mapping of cortical language areas in adults. Intraoperative approaches. Adv Neurol 63:155163,1993

31. Perrine K, Devinsky O, Uysal S, Santschi C, Doyle WK: Cortical mapping of right hemisphere functions. Epilepsy Behav 1:7-16, 2000

32. Roux FE, Dufor O, Lauwers-Cances V, Boukhatem L, Brauge D, Draper L, et al: Electrostimulation mapping of spatial neglect. Neurosurgery 69:1218-1231, 2011

33. Sanai N, Berger MS: Extent of resection influences outcomes for patients with gliomas. Rev Neurol (Paris) 167:648-654, 2011

34. Sanai N, Chang S, Berger MS: Low-grade gliomas in adults. J Neurosurg 115:948-965, 2011

35. Sanai N, Mirzadeh Z, Berger MS: Functional outcome after language mapping for glioma resection. $\mathbf{N}$ Engl J Med 358:18-27, 2008

36. Sanai N, Polley MY, Berger MS: Insular glioma resection: assessment of patient morbidity, survival, and tumor progression. J Neurosurg 112:1-9, 2010

37. Sanai N, Polley MY, McDermott MW, Parsa AT, Berger MS: An extent of resection threshold for newly diagnosed glioblastomas. J Neurosurg 115:3-8, 2011

38. Skrap M, Mondani M, Tomasino B, Weis L, Budai R, Pauletto G, et al: Surgery of insular nonenhancing gliomas: volumetric analysis of tumoral resection, clinical outcome, and survival in a consecutive series of 66 cases. Neurosurgery 70:1081-1094, 2012

39. Smith JS, Chang EF, Lamborn KR, Chang SM, Prados MD, Cha S, et al: Role of extent of resection in the long-term 
outcome of low-grade hemispheric gliomas. J Clin Oncol 26:1338-1345, 2008

40. Szelényi A, Bello L, Duffau H, Fava E, Feigl GC, Galanda $\mathrm{M}$, et al: Intraoperative electrical stimulation in awake craniotomy: methodological aspects of current practice. Neurosurg Focus 28(2):E7, 2010

41. Thiebaut de Schotten M, Urbanski M, Duffau H, Volle E, Lévy R, Dubois B, et al: Direct evidence for a parietal-frontal pathway subserving spatial awareness in humans. Science 309:2226-2228, 2005

42. Tomasino B, Marin D, Canderan C, Maieron M, Budai R, Fabbro F, et al: Involuntary switching into the native language induced by electrocortical stimulation of the superior temporal gyrus: a multimodal mapping study. Neuropsychologia 62:87-100, 2014

43. Tomasino B, Marin D, Maieron M, Ius T, Budai R, Fabbro F, et al: Foreign accent syndrome: a multimodal mapping study. Cortex 49:18-39, 2013

44. Wager M, Du Boisgueheneuc F, Pluchon C, Bouyer C, Stal V, Bataille B, et al: Intraoperative monitoring of an aspect of executive functions: administration of the Stroop test in 9 adult patients during awake surgery for resection of frontal glioma Neurosurgery 72 (2 Suppl Operative):ons169-ons181, 2013

\section{Disclosures}

The authors report no conflict of interest concerning the materi- als or methods used in this study or the findings specified in this paper.

\section{Author Contributions}

Conception and design: Tomasino, Skrap, Marin. Acquisition of data: Tomasino, Skrap, Marin, Ius. Analysis and interpretation of data: all authors. Drafting the article: Tomasino, Skrap, Marin. Critically revising the article: all authors. Approved the final version of the manuscript on behalf of all authors: Tomasino. Statistical analysis: Tomasino, Marin, Ius. Study supervision: Tomasino, Skrap.

\section{Supplemental Information}

Online-Only Content

Supplemental material is available with the online version of the article.

Supplementary Tables 1-3. http://thejns.org/doi/suppl/10.3171/ 2015.10.JNS15740

\section{Correspondence}

Barbara Tomasino, IRCCS “E. Medea," Polo Regionale del Friuli Venezia Giulia, Via della Bontà, 7, San Vito al Tagliamento (PN) 33078, Italy. email: barbara.tomasino@gmail.com. 